$08.2 ; 01.1$

\title{
Кинетика самопроизвольного формирования структуры ядро-оболочка в нитевидных нанокристаллах (In,Ga)As
}

\author{
() Н.В. Сибирев ${ }^{1}$, Ю.С. Бердников ${ }^{1-3}$, И.В. Штром ${ }^{1,4}$, Е.В. Убыйвовк ${ }^{1,2}$, Р.Р. Резник ${ }^{1,2,5}$, Г.Э. Цырлин ${ }^{1,2,4}$ \\ ${ }^{1}$ Санкт-Петербургский государственный университет, Санкт-Петербург, Россия \\ ${ }^{2}$ Санкт-Петербургский национальный исследовательский Академический университет им. Ж.И. Алфёрова РАН, \\ Санкт-Петербург, Россия \\ ${ }^{3}$ Национальный исследовательский университет „Высшая школа экономики“, Санкт-Петербург, Россия \\ ${ }^{4}$ Институт аналитического приборостроения РАН, Санкт-Петербург, Россия \\ ${ }^{5}$ Университет ИТМО, Санкт-Петербург, Россия \\ E-mail: N.Sibirev@spbu.ru
}

Поступило в Редакцию 13 мая 2021 г.

В окончательной редакции 21 октября 2021г.

Принято к публикации 22 октября 2021 г.

Предложена модель самопроизвольного формирования структуры ядро-оболочка в ходе аксиального роста нитевидных нанокристаллов (In,Ga)As при синтезе методом молекулярно-пучковой эпитаксии без независимого бокового роста. В рамках предложенной модели рассчитано распределение In поперек оси нитевидного нанокристалла, согласующееся с экспериментальными наблюдениями.

Ключевые слова: нитевидные нанокристаллы тройного состава, структуры ядро-оболочка, нитевидные нанокристаллы (In,Ga)As, радиальные гетероструктуры.

DOI: 10.21883/PJTF.2022.03.51980.18869

Нитевидные нанокристаллы (ННК) III-V полупроводниковых соединений находят применение в качестве базовых элементов для формирования активных областей целого ряда новых оптоэлектронных устройств [1-3]. Развитая боковая поверхность ННК обусловливает применение их в сенсорике [2,3], а также позволяет совмещать материалы, сильно различающиеся по параметру решетки, в одной структуре типа ядро-оболочка [3].

Молекулярно-пучковая эпитаксия (МПЭ) в настоящее время является одним из самых распространенных методов роста III-V ННК [2,3]. При синтезе ННК со структурой ядро-оболочка, как правило, рост ядра осуществляется по механизму пар-жидкость-кристалл или пар-кристалл-кристалл с жидкой каплей или твердой частицей катализатора на вершине. При этом оболочка формируется в результате независимого осаждения материала по механизму пар-кристалл на боковых стенках ННК [4]. В такого рода ростовых процессах неравномерность бокового роста вдоль оси ННК приводит к формированию сужающихся к вершине структур [4]. Кроме того, экспериментально было показано, что уровень легирования при росте ядра по механизму пар-жидкость-кристалл, а оболочки по механизму пар-кристалл может различаться на порядок [5].

В настоящей работе предлагается альтернативная модель формирования НHK (In, Ga)As со структурой ядро-оболочка в рамках одного процесса без независимого радиального роста. Впервые предложена модель, объясняющая механизм формирования таких ННК в ходе МПЭ-роста, проведено сравнение модельных результатов с экспериментальными.
Обычно рост ННК проходит послойно [6], а состав каждого монослоя (МС) определяется скоростями встраивания компонентов в движущуюся ступень формирующегося МC [7,8]. Для записи данного эффекта используются разные формулы [6-9], здесь мы воспользуемся формулой из работы [8]:

$$
x=\frac{v_{\mathrm{InAs}}}{v_{\mathrm{InAs}}+v_{\mathrm{GaAs}}},
$$

где $v_{\mathrm{InAs}}$ и $v_{\mathrm{GaAs}}-$ скорости встраивания пар атомов In-As и $\mathrm{Ga}-\mathrm{As}$ в ступень.

В предположении малого размера критического зародыша нового МC $[9,10]$ скорость встраивания пар $\mathrm{In}-\mathrm{As}$ и $\mathrm{Ga}-\mathrm{As}$ в ступень может быть описана формулами $[11,12]$ :

$$
\begin{gathered}
v_{\mathrm{InAs}}=k_{\mathrm{InAs}} C_{\mathrm{As}} C_{\mathrm{In}}, \\
v_{\mathrm{GaAs}}=k_{\mathrm{GaAs}} C_{\mathrm{As}} C_{\mathrm{Ga}} .
\end{gathered}
$$

Здесь $C_{\mathrm{As}}, C_{\mathrm{Ga}}, C_{\mathrm{In}}-$ соответственно концентрации мышьяка, галлия и индия в капле катализатора, $k_{\operatorname{InAs}}$ и $k_{\mathrm{GaAs}}$ - кинетические коэффициенты.

Для дальнейшего анализа введем обозначения для безразмерных величин: $k=\frac{k_{\operatorname{InAs}}}{k_{\operatorname{InAs}}+k_{\mathrm{GaAs}}}$, $y=\frac{C_{\text {In }}}{C_{\mathrm{Ga}}+C_{\mathrm{In}}}=\frac{N_{\mathrm{In}}}{N_{\mathrm{Ga}}+N_{\mathrm{In}}}$, где $N_{\mathrm{In}}$ и $N_{\mathrm{Ga}}$ - число атомов индия и галлия В катализаторе соответственно. В терминах этих обозначений, согласно (1), связь между составом катализатора $y$ и составом движущейся ступени $x$ представляется как

$$
x=\frac{k y}{2 k y-k-y+1} .
$$



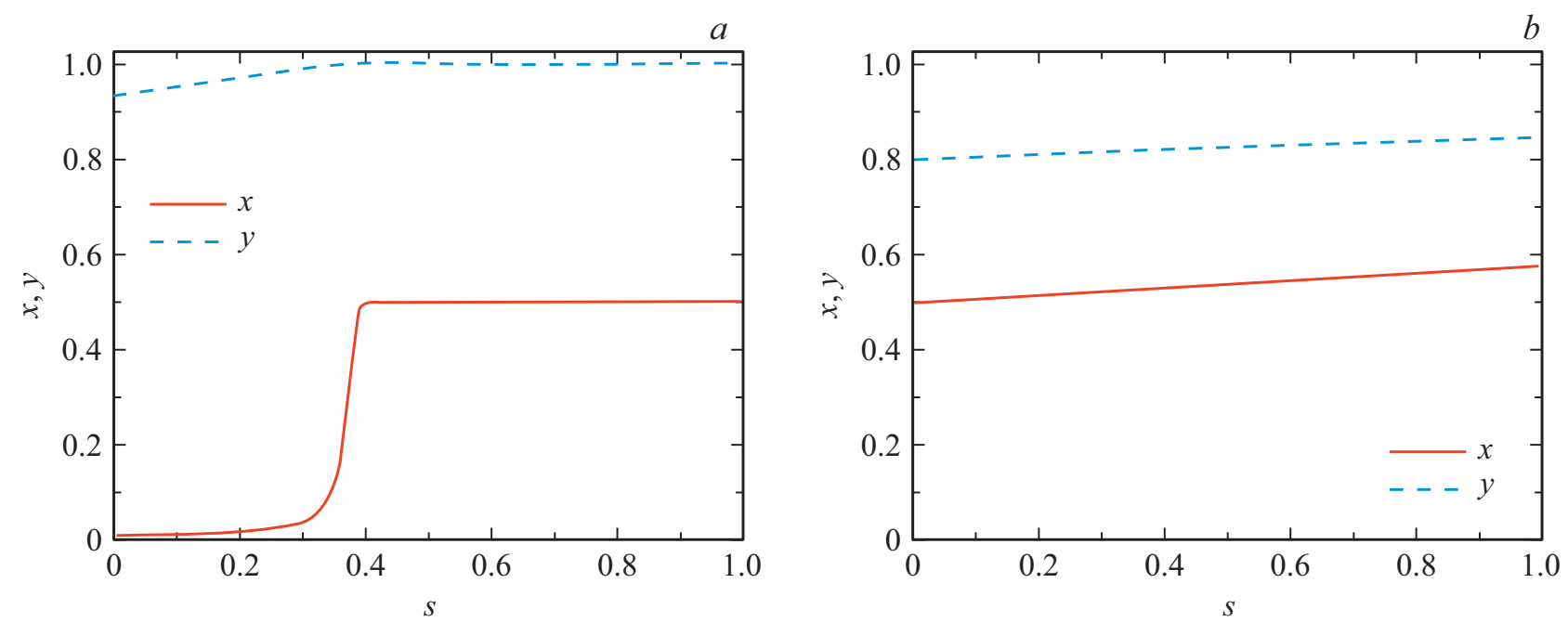

Рис. 1. Изменение состава ступени $(x)$ и состава капли $(y)$ по мере заполнения монослоя $(s) . a-$ для параметров $k=0.00045$, $N_{0}=3, z=0.3, f=1.4, y_{0}=0.94 ; b-$ для параметров $k=0.2, N_{0}=3, z=0.5, f=2, y_{0}=0.8$.

При МПЭ-росте НHК (In,Ga)As число атомов III группы в катализаторе $N$ задается суммарными потоками атомов In $\left(F_{\mathrm{In}}\right)$ и $\mathrm{Ga}\left(F_{\mathrm{Ga}}\right)$ и скоростью встраивания атомов в ступень нового МС. Для удобства записи уравнений количество атомов в капле $N, N_{0}, N_{\text {In }}$ и $N_{\mathrm{Ga}}$ будем выражать в МС. При этом скорость движения ступени лимитируется поступлением Аs из газовой среды, поток которого обозначим как $F_{\mathrm{As}}$, в силу более низкой растворимости As в капле катализатора в сравнении с In и Ga $[1,13]$. В терминах $f=\frac{F_{\mathrm{As}}}{F_{\mathrm{In}}+F_{\mathrm{Ga}}}, z=\frac{F_{\mathrm{In}}}{F_{\mathrm{Ga}}+F_{\mathrm{In}}}$ уравнение для $N$ принимает вид

$$
\frac{d N}{d s}=\frac{1}{f}-1
$$

где $s=F_{\mathrm{As}} t-$ заполненность верхней грани ННК, изменяющаяся в пределах от 0 до 1. Уравнение для количества In в капле принимает вид

$$
\frac{d(y N)}{d s}=\frac{z}{f}-x
$$

Обозначим количество атомов III группы в катализаторе в момент зарождения нового $\mathrm{MC}(s=0)$ как $N_{0}$. Тогда уравнение (6) с учетом (4) может быть записано в виде

$$
\begin{aligned}
& \frac{(1-2 k) y-1+k}{((1-2 k) y-1+k)\left(y+\frac{z}{f-1}\right)+\frac{f k}{f-1} y} \frac{d y}{d s} \\
& =\frac{1}{\frac{f}{f-1} N_{0}-s} .
\end{aligned}
$$

Вместе с начальным условием $y(0)=y_{0}$ уравнения (4) и (7) описывают динамику изменения состава капли и состава формирующегося МС.

Типичные примеры зависимостей составов катализатора и растущего МС представлены на рис. 1. Рис. 1, иллюстрирует ситуацию, когда концентрация в капле быстрокристаллизующегося элемента (Ga) экспоненциально быстро падает при росте МС. В этом случае в начале формирования MC In почти не встраивался в ННК (т.е. $x \approx 0$ ). По мере формирования МС доля хорошо растворимого в капле элемента (In) растет, пока не становится почти равной единице (т.е. $y \sim 1)$. После этого на завершающей стадии формирования МС его состав определяется поступлением быстрокристаллизующегося элемента $(\mathrm{Ga})$, и $x \approx 1-\frac{1-z}{f}$. Для сравнения на рис. $1, b$ представлена ситуация, когда плохо растворимый, но быстро кристаллизующийся элемент $(\mathrm{Ga})$ не успевает израсходоваться при формировании МС.

Для расчета профиля состава в сечении ННК необходимо задаться вопросом о месте возникновения зародыша и его форме. Основываясь на данных in situ наблюдений роста отдельных МС в ННК, мы предполагаем, что зародыш нового слоя образуется в углу верхней грани ННК и имеет форму треугольника $[9,10]$ (см. вставку на рис. 2,a). Поскольку образование зародыша равновероятно в каждом из шести углов верхней грани, для получения профиля ННК по составу необходимо провести усреднение по шести углам.

Для такой геометрии зародыша заполненность МС после усреднения по всем вершинам ННК будет одинаковой для всех точек внутри шестиугольника и равной $1 / 2$. Поэтому линейная зависимость состава ступени от заполненности $x(s)$ (пример зависимости на рис. $1, b$ ) будет приводить к однородному составу в поперечном сечении ННК.

Для образования неоднородного в поперечном сечении ННК необходима сильно нелинейная зависимость состава ступени от заполненности (см., например, зависимость на рис. 1,a). На рис. 2, a приведен пример неоднородного распределения доли In в поперечном сечении ННК. В центральной части (ядре) ННК 

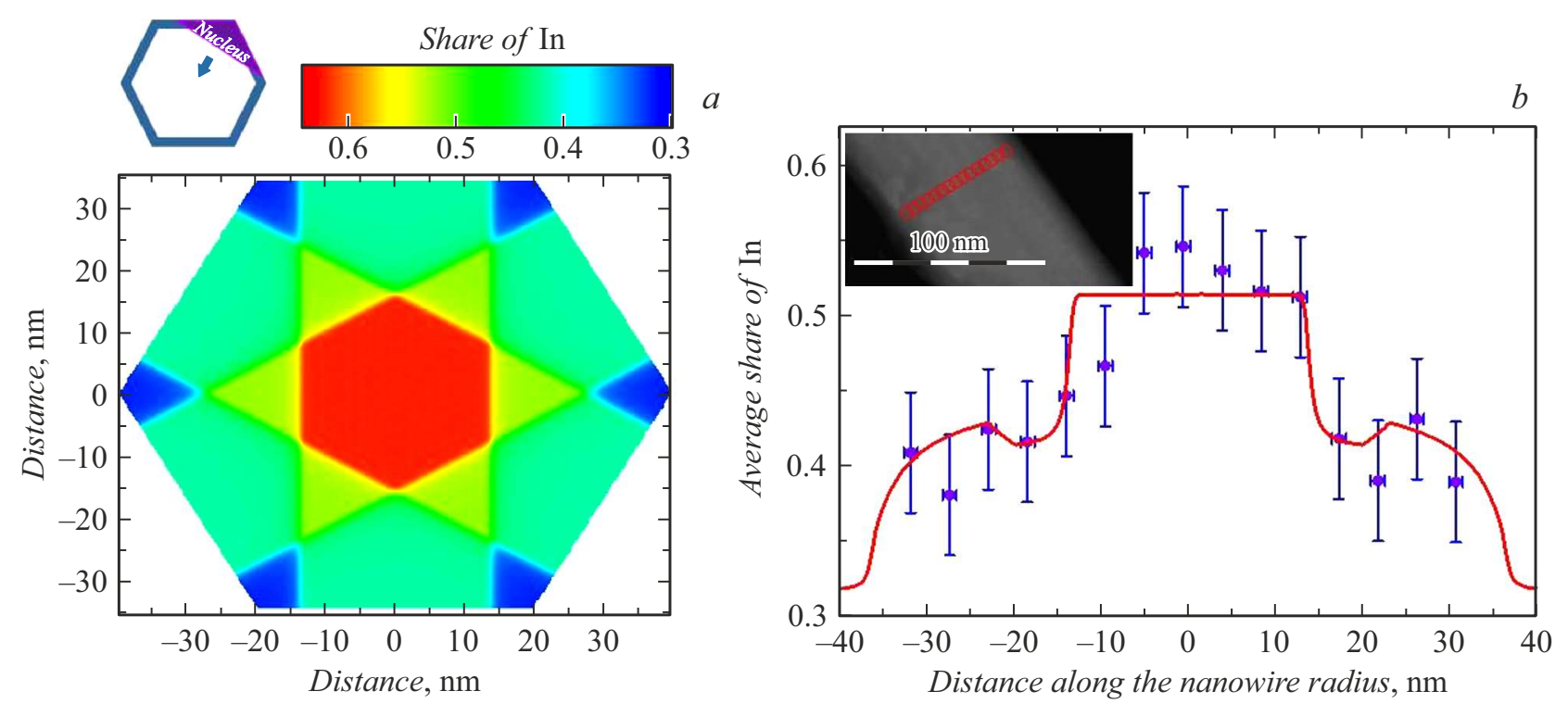

Рис. 2. $a-$ расчет профиля состава в поперечном сечении ННК; $b$ - профиль состава поперек оси ННК, точки экспериментальные измерения, сплошная линия - расчет с параметрами $z=0.46, N_{0}=2.4, f=1.42, k=5.4 \cdot 10^{-4}, y_{0}=0.96$.

(In, Ga)As концентрация In выше, чем в периферийной части (оболочке). Таким образом, обеднение капли катализатора по $\mathrm{Ga}$ может приводить к формированию структуры ядро-оболочка в ходе роста по механизму пар-жидкость-кристалл. В более общем случае в зависимости от параметров роста оболочка может быть обогащена как плохо растворимым в капле $\mathrm{Ga}$, так и легкорастворимым In.

При расчете профиля состава ННК, приведенного на рис. $2, a$, использовались значения параметров, отвечающие условиям экспериментов по МПЭ-росту ННК $(\mathrm{In}, \mathrm{Ga})$ As. Рост НHК с золотым катализатором осуществлялся на подложках $\mathrm{Si}(111)$ в течение $20 \mathrm{~min}$ в As-стабилизированных условиях, поэтому для моделирования подбиралось значение отношения потоков V/III групп в диапазоне $f=1-1.5$. Эффективная скорость осаждения и температура роста поддерживались постоянными и составляли $0.8 \mathrm{ML} / \mathrm{s}$ и $220^{\circ} \mathrm{C}$ соответственно.

Профиль состава поперек оси ННК был измерен на просвечивающем электронном микроскопе (ПЭМ) Zeiss Libra 200FE, оснащенном энергодисперсионным детектором рентгеновского излучения (EDX) Oxford $\mathrm{X}$-Мах 80. Пример ПЭМ-изображения ННК и результаты измерения состава поперек оси ННК представлены на рис. $2, b$. Экспериментально наблюдается обогащение центральной части ННК индием. Во внутренней трети ННК доля In составляет около $50 \%$, во внешней части около $40 \%$. Обогащение оболочки $\mathrm{Ga}$ может объясняться его более активным в сравнении с In встраиванием на начальном этапе формирования MC.

Известно, что при самокаталитическом росте ННК $(\mathrm{In}, \mathrm{Ga})$ As концентрация $\mathrm{Ga}$ в капле остается на пределе чувствительности EDX-детекторов (менее 5\%), даже когда доля $\mathrm{Ga}$ в ННК достигает 80-90\% [14], что позволяет оценить $k<0.002$.

После остановки роста на вершинах ННК наблюдались капли золота радиусом менее $5 \mathrm{~nm}$, что позволяет оценить концентрацию золота в капле во время роста. Диаметр капли во время роста примерно равен диаметру ННК, т.е. более $50 \mathrm{~nm}$. Если высота капли во время роста оставалась неизменной, то концентрацию золота можно оценить как отношение квадратов диаметра замерзшей капли и диаметра ННК, т.е. менее $1 \%$ в нашем случае. Поэтому капля почти полностью состоит из In и $\mathrm{Ga}$, температуры плавления которых ниже температуры роста.

Более точные значения параметров были получены при аппроксимации измерений состава поперек оси ННК (рис. $2, b$ ) модельной зависимостью. Для этого расчетное распределение In в поперечном сечении ННК (рис. 2,a) было усреднено вдоль оси, перпендикулярной направлению сканирования. Параметр $z=0.46$ был найден как средний состав ННК. Далее методом градиентного спуска подбирались значения модельных параметров. При $k=0.00054, N_{0}=2.4, f=1.42, y_{0}=0.96$ было получено наилучшее совпадение расчетной и экспериментальной зависимостей. В момент нуклеации $\mathrm{MC}$ доля $\mathrm{Ga}$ в капле максимальна (около 4\%), но во время роста MC быстро падает до менее чем $0.1 \%$ и остается такой до завершения МC.

В заключение отметим, что в работе представлена модель, объясняющая спонтанное образование радиальной структуры ядро-оболочка в $\mathrm{HHK}(\mathrm{In}, \mathrm{Ga}) \mathrm{As}$. Расчетные профили состава ННК хорошо согласуются с экспериментальными данными, полученными в ходе МПЭ-роста ННК. 


\section{Финансирование работы}

Рост ННК и моделирование выполнены при финансовой поддержке Российского научного фонда (проект № 18-72-10047). Диагностика ННК проведена с использованием оборудования Междисциплинарного ресурсного центра СПбГУ по направлению „Нанотехнологии“ при поддержке гранта Санкт-Петербургского университета № 75746688. Р.Р. Резник благодарит за поддержку Министерство науки и высшего образования РФ, проект тематики научных исследований № 2019-1442. Ю.С. Бердников благодарит НИУ ВШЭ за поддержку в рамках Программы фундаментальных исследований в 2021 г.

\section{Конфликт интересов}

Авторы заявляют, что у них нет конфликта интересов.

\section{Список литературы}

[1] В.Г. Дубровский, Г.Э. Цырлин, В.М. Устинов, ФТП, 43 (12), 1585 (2009). [V.G. Dubrovskii, G.E. Cirlin, V.M. Ustinov, Semiconductors, 43 (12), 1539 (2009). DOI: $10.1134 / \mathrm{S} 106378260912001 \mathrm{X}]$.

[2] E. Barrigón, M. Heurlin, Z. Bi, B. Monemar, L. Samuelson, Chem. Rev., 119, 9170 (2019). DOI: 10.1021/acs.chemrev.9b00075

[3] M. Royo, M. De Luca, R. Rurali, I. Zardo, J. Phys. D: Appl. Phys., 50, 143001 (2017). DOI: 10.1088/1361-6463/aa5d8e

[4] V.G. Dubrovskii, I.V. Shtrom, R.R. Reznik, Y.B. Samsonenko, A.I. Khrebtov, I.P. Soshnikov, S. Rouvimov, N. Akopian, T. Kasama, G.E. Cirlin, Cryst. Growth Des., 16, 7251 (2016). DOI: $10.1021 /$ acs.cgd.6b01412

[5] E. Dimakis, M. Ramsteiner, A. Tahraoui, H. Riechert, L. Geelhaar, Nano Res., 5, 796 (2012). DOI: $10.1007 / \mathrm{s} 12274-012-0263-9$

[6] В.Г. Дубровский, Н.В. Сибирев, Г.Э. Цырлин, Письма в ЖТФ, 30 (16), 41 (2004). [V.G. Dubrovskii, N.V. Sibirev, G.E. Cirlin, Tech. Phys. Lett., 30 (8), 682 (2004). DOI: $10.1134 / 1.1792313]$.

[7] P. Periwal, N.V. Sibirev, G. Patriarche, B. Salem, F. Bassani, V.G. Dubrovskii, T. Baron, Nano Lett., 14, 5140 (2014). DOI: $10.1021 / \mathrm{nl} 5019707$

[8] J. Johansson, M. Ghasemi, Phys. Rev. Mater., 1, 040401 (2017). DOI: 10.1103/PhysRevMaterials.1.040401

[9] C.B. Maliakkal, E.K. Mårtensson, M.U. Tornberg, D. Jacobsson, A.R. Persson, J. Johansson, L.R. Wallenberg, K.A. Dick, ACS Nano., 14, 3868 (2020). DOI: $10.1021 /$ acsnano.9b09816

[10] J.C. Harmand, G. Patriarche, F. Glas, F. Panciera, I. Florea, J.-L. Maurice, L. Travers, Y. Ollivier, Phys. Rev. Lett. 121, 166101 (2018). DOI: 10.1103/PhysRevLett.121.166101

[11] Н.В. Сибирев, Письма в ЖТФ, 41 (5), 1 (2015). [N.V. Sibirev, Tech. Phys. Lett., 41 (3), 209 (2015). DOI: $10.1134 / \mathrm{S} 1063785015030153]$.

[12] В.Г. Дубровский, Письма в ЖТФ, 42 (6), 104 (2016). [V.G. Dubrovskii, Tech. Phys. Lett., 42 (3), 332 (2016). DOI: $10.1134 / \mathrm{S} 1063785016030196]$.
[13] P. Krogstrup, H.I. Jørgensen, E. Johnson, M.H. Madsen, C.B. Sørensen, A.F.I. Morral, M. Aagesen, J. Nygárd, F. Glas, J. Phys. D: Appl. Phys., 46 (31), 313001 (2013). DOI: $10.1088 / 0022-3727 / 46 / 31 / 313001$

[14] A. Scaccabarozzi, A. Cattoni, G. Patriarche, L. Travers, S. Collin, J.C. Harmand, F. Glas, F. Oehler, Nanoscale, 12, 18240 (2020). DOI: 10.1039/d0nr04139d 